Bull. Korean Math. Soc. 52 (2015), No. 4, pp. 1285-1295

http://dx.doi.org/10.4134/BKMS.2015.52.4.1285

\title{
THE STRONG MORI PROPERTY IN RINGS WITH ZERO DIVISORS
}

\author{
Dechuan Zhou and Fanggui Wang
}

\begin{abstract}
An SM domain is an integral domain which satisfies the ascending chain condition on $w$-ideals. Then an SM domain also satisfies the descending chain condition on those chains of $v$-ideals whose intersection is not zero. In this paper, a study is begun to extend these properties to commutative rings with zero divisors. A $Q_{0}-\mathrm{SM}$ ring is defined to be a ring which satisfies the ascending chain condition on semiregular $w$-ideals and satisfies the descending chain condition on those chains of semiregular $v$-ideals whose intersection is semiregular. In this paper, some properties of $Q_{0}$-SM rings are discussed and examples are provided to show the difference between $Q_{0}$-SM rings and SM rings and the difference between $Q_{0}$-SM rings and $Q_{0}$-Mori rings.
\end{abstract}

\section{Introduction}

In this paper, we assume that $R$ is a commutative ring with an identity and $S$ is a multiplicatively closed set of $R$. Let $Z(R)$ denote the set of zero divisors of $R$ and let $T(R)$ be the total quotient ring of $R$.

Let $I$ be an ideal of $R$. Then $I$ is called a regular ideal if $I$ contains a regular element and $I$ is called a semiregular ideal of $R$ if $I$ contains a finitely generated ideal $I_{0}$ which has no nonzero annihilator. Let $S^{0}$ denote the set of finitely generated semiregular ideals. T. Lucas used semiregular ideals to define the ring of finite fractions $Q_{0}(R)$ and tried to use $Q_{0}(R)$ instead of the quotient field of a domain to research multiplicative ideal theory of commutative rings with zero divisors, where

$$
Q_{0}(R)=\left\{u \in T(R[X]) \mid I u \subseteq R \text { for some } I \in S^{0}\right\} .
$$

If $u$ is an element of $Q_{0}(R)$, then $u$ can be written in the form $u=\frac{\sum_{i=0}^{n} a_{i} X^{i}}{\sum_{i=0}^{n} b_{i} X^{i}} \in$ $T(R[X])$ with $a_{i} b_{j}=a_{j} b_{i}$ for each $i$ and $j$. If each semiregular ideal of $R$ is regular, then $Q_{0}(R)=T(R)$.

Received September 1, 2014; Revised October 23, 2014.

2010 Mathematics Subject Classification. 13A15, $13 \mathrm{~F} 05$.

Key words and phrases. $Q_{0}-\mathrm{SM}$ ring, semiregular $w$-ideal, semiregular $v$-ideal.

Supported by the National Natural Science Foundation of China(Grant No.11171240). 
For a domain $D$ with the quotient field $K$, an ideal $I$ of $D$ is called a $v$ ideal if $\left(I^{-1}\right)^{-1}=I$, where $I^{-1}=\{x \in K \mid x I \subseteq R\}$. A Mori domain, i.e., a domain which satisfies the ascending chain condition on $v$-ideals, has many good properties and various researchers have focused on it. The definition of Mori domains is analogous to that of Noetherian domains, but many classical theorems on Noetherian domains cannot be generalized to Mori domains. In order to give a description in the star operation theory corresponding to the classical theory of Noetherian domains, in 1997, Wang and McCasland gave the definitions of the $w$-operation and SM domains (strong Mori domains) [8, Definition 2 and Definition 4]. Let $J$ be a finitely generated ideal of a domain $D$. If $J^{-1}=D$, then $J$ is called a $G V$-ideal, denoted by $J \in \mathrm{GV}(D)$. A torsion-free module $M$ is called a $w$-module of $D$ if whenever $J x \subseteq M$ for some $J \in \operatorname{GV}(D)$ and $x \in K \bigotimes_{D} M$, then $x \in M$. An SM domain is an integral domain which satisfies the ascending chain condition on $w$-ideals. Wang and McCasland also have shown that SM domains satisfy the Principal Ideal Theorem [9, Corollary 1.11], the Hilbert Basis Theorem [9, Theorem 1.13] and the Krull Intersection Theorem [9, Theorem 1.8] (Note that these three theorems do not hold for Mori domains in general).

In 2004, T. Lucas introduced the $Q_{0}$-Mori ring and discussed the Mori property in commutative rings with zero divisors. The $Q_{0}$-Mori ring is defined to be a ring which satisfies ACC (i.e., the ascending chain condition) on semiregular $v$-ideals and satisfies the restricted DCC on semiregular $v$-ideals (i.e., the descending chain condition on those chains of semiregular $v$-ideals whose intersection is semiregular) (see [5, page 1]), where a semiregular $v$-ideal is not only a semiregular ideal but also a $v$-ideal. Following his idea, in this paper, the definition of $Q_{0}$-SM rings is provided and we use $Q_{0}(R)$ to discuss the SM property in commutative rings with zero divisors.

\section{The reducible property of commutative rings}

We say that an $R$-module $B \subseteq Q_{0}(R)$ is a fractional ideal of $R$ if there is a semiregular ideal $I \subseteq R$ such that $I B \subseteq R$. If, in addition, $B$ contains an element that is not a zero divisor and there is a regular element $r \in R$ such that $r B \subseteq R$, then it is a regular fractional ideal of $R$. For $B$ to be considered a semiregular fractional ideal, $B$ must contain a finitely generated fractional ideal that has no nonzero annihilator and there must be a semiregular ideal $I$ of $R$ such that $I B \subseteq R$.

We let

$$
R_{\langle S\rangle}=\left\{q \in Q_{0}(R) \mid r q \in R \text { for some } r \in S\right\} .
$$

It is clear that $R_{\langle S\rangle}$ is a ring and we call $R_{\langle S\rangle}$ the $Q_{0}$-quotient ring with respect to $S$. For an ideal $I$ of $R$, we set

$$
I_{\langle S\rangle}=\left\{q \in R_{\langle S\rangle} \mid r q \in I \text { for some } r \in S\right\} .
$$


For a prime ideal $P$ of $R, S=R \backslash P$ is a multiplicatively closed set, then we consider $R_{\langle S\rangle}$ as $R_{\langle P\rangle}$ and consider $I_{\langle S\rangle}$ as $I_{\langle P\rangle}$. Here we call $R_{\langle P\rangle}$ the $Q_{0^{-}}$ quotient ring with respect to $P$. It is easy to see that if $I$ is not contained in $P$, then $I_{\langle P\rangle}=R_{\langle P\rangle}$.

Definition 2.1. Let $A \subseteq Q_{0}(R)$ be an $R$-module. Then $A$ is called an $S$ reducible module if whenever $s x \in A$ where $s \in S$ and $x \in Q_{0}(R)$, we have $x \in A$. For a prime ideal $P$ of $R$ and $S=R \backslash P$, we say that $A$ is $P$-reducible if $A$ is $S$-reducible.

For $R$ and $S, R$ may not be its own $S$-reducible ideal. For example, let $R$ be the ring of integers $\mathbb{Z}$ and $S=R \backslash\{0\}$. Then $Q_{0}(R)=\mathbb{Q}$ where $\mathbb{Q}$ is the rational number field. We choose $s \in S$ and $1 / s \in Q_{0}(R)$, then $s \cdot \frac{1}{s}=1 \in R$, but $1 / s \notin R$. We also have that not all $R_{\langle S\rangle}$-modules are $S$-reducible. For example, let $s \in R, s \neq 0$ and $s^{2}=0$. Then $S=\{0,1, s\}$ is a multiplicatively closed set of $R$. Clearly, $C=0$ is an $R_{\langle S\rangle}$-module. Since $s s=0 \in C$ and $s \notin C$, we have $C$ is not $S$-reducible.

Lemma 2.2. Let $S$ be a multiplicatively closed set in $R$.

(1) Let $A$ be an ideal of $R_{\langle S\rangle}$ and let $B=A \cap R$. Then $A \subseteq B_{\langle S\rangle}$.

(2) $I$ is a proper $S$-reducible ideal of $R_{\langle S\rangle}$ if and only if $I=I_{\langle S\rangle}=(I \cap R)_{\langle S\rangle}$ and $I \cap S=\emptyset$.

(3) If $A$ is a proper ideal of $R$, then $A_{\langle S\rangle}$ is an $S$-reducible ideal of $R_{\langle S\rangle}$ and $A_{\langle S\rangle}=\left(A_{\langle S\rangle}\right)_{\langle S\rangle}$.

In order to discuss the relationship between reducible ideals and $w$-ideals, we need to know the concept of $w$-modules of commutative rings in [10]. Let $J$ be a finitely generated ideal of $R$. If the natural homomorphism $\varphi: R \rightarrow$ $J^{*}=\operatorname{Hom}_{R}(J, R)$ is an isomorphism, then $J$ is called a $G V$-ideal, denoted by $J \in \mathrm{GV}(R)$. An $R$-module $M$ is called a $G V$-torsion-free module if whenever $J x=0$ for some $J \in \mathrm{GV}(R)$ and $x \in M$, we have $x=0$. A GV-torsion-free module $M$ is called a $w$-module if $\operatorname{Ext}_{R}^{1}(R / J, M)=0$ for each $J \in \operatorname{GV}(R)$ and the $w$-envelope of $M$ is the set given by

$$
M_{w}=\{x \in E(M) \mid J x \subseteq M \text { for some } J \in \mathrm{GV}(R)\},
$$

where $E(M)$ is the injective hull of $M$. Therefore $M$ is a $w$-module if and only if $M_{w}=M$. In this paper, let $w$-Max $(R)$ denote the set of all maximal $w$-ideals of $R$. For $R$, there is at least one maximal $w$-ideal and each maximal $w$-ideal is prime.

Lemma 2.3. Let $P$ be a prime $w$-ideal of $R$. If $I$ is a P-reducible ideal of $R_{\langle P\rangle}$, then $I$ is a $w$-module of $R$.

Proof. Let $x \in I_{w}$. Then there is some $J \in \mathrm{GV}(R)$ such that $J x \subseteq I$. Since $J \nsubseteq P$, there is some $s \in J \backslash P$ such that $s x \in I$. Because $I_{w} \subseteq\left(Q_{0}(R)\right)_{w}=$ $Q_{0}(R)$ and $I$ is $P$-reducible, we have $x \in I$. Thus $I$ is a $w$-module of $R$. 
Theorem 2.4. Let $P$ be a prime $w$-ideal of $R$. If $R$ satisfies $A C C$ on semiregular $w$-ideals, then $R_{\langle P\rangle}$ satisfies $A C C$ on semiregular $P$-reducible ideals.

Proof. Let $I$ be a semiregular $P$-reducible ideal of $R_{\langle P\rangle}$ and let $A=I \cap R$. We first show that $A$ is a semiregular $w$-ideal of $R$. Let $B=\left(b_{1}, b_{2}, \ldots, b_{n}\right) \subseteq I$ be a finitely generated semiregular ideal of $R_{\langle P\rangle}$. Then for each $i$ there is a finitely generated semiregular ideal $I_{\alpha_{i}}$ of $R$ such that $b_{i} I_{\alpha_{i}} \subseteq R$. Let $I_{0}=$ $I_{\alpha_{1}} I_{\alpha_{2}} \cdots I_{\alpha_{n}}$. Then $I_{0}$ is also a semiregular ideal of $R$ and $B I_{0} \subseteq I \cap R=$ $A$. Since $B I_{0}$ is a finitely generated semiregular ideal of $R$, we have $A$ is semiregular. By Lemma 2.3, $I$ is a $w$-module of $R$. We have $A$ is a $w$-ideal of $R$.

Let $\left\{I_{n}\right\}$ be an ascending chain of semiregular $P$-reducible ideals of $R_{\langle P\rangle}$. Then $\left\{I_{n} \cap R\right\}$ is an ascending chain of semiregular $w$-ideals of $R$. Let $A_{i}=$ $I_{i} \cap R$. There is an integer $n$ such that $I_{k} \cap R=I_{n} \cap R$ for each $k \geq n$. So $\left(A_{k}\right)_{\langle P\rangle}=\left(A_{n}\right)_{\langle P\rangle}$. Because $I_{i}$ is a $P$-reducible ideal, $I_{i}=\left(I_{i} \cap R\right)_{\langle P\rangle}=$ $\left(A_{i}\right)_{\langle P\rangle}$ by Lemma $2.2(2)$. Then $I_{k}=I_{n}$. It follows that $R_{\langle P\rangle}$ satisfies ACC on semiregular $P$-reducible ideals.

For a semiregular fractional ideal $I$, let $I^{-1}=(R: I)=\left\{t \in Q_{0}(R) \mid t I \subseteq R\right\}$ and let $I_{v}=\left(I^{-1}\right)^{-1}$. Then it is easy to show that $(R: I)$ is a semiregular fractional ideal as well and $(R: I)=(R: I)_{v}$. Set $I_{t}=\bigcup\left(I_{0}\right)_{v}$ with the union taken over the finitely generated fractional ideals that are contained in $I$ (see [5, Introduction]). If $I=I_{v}$ (resp., $I=I_{t}$ ), then $I$ is said to be a $v$-fractional ideal (resp., $t$-fractional ideal). For each semiregular fractional ideal $I$, we have $I_{w} \subseteq I_{t} \subseteq I_{v}$ and if $I$ is finitely generated, we have $I_{v}=I_{t}$.

For a finitely generated semiregular ideal $J$ of $R, J$ is a GV-ideal if and only if $J^{-1}=R$. Let $M$ be a semiregular prime ideal of $R$. Then $M$ is a maximal $w$-ideal if and only if $M$ is a maximal $t$-ideal. We use $t$-Max $(R)$ to denote the set of all maximal $t$-ideals of $R$.

Lemma 2.5 ([5, Lemma 2.15]). Let I be a semiregular ideal of $R$.

(1) If $I$ is a $v$-ideal, then $I=\bigcap\left\{\left(I_{\langle M\rangle}\right)_{v} \mid M \in t\right.$-Max $\left.(R)\right\}$.

(2) If I is finitely generated, then $I_{v}=\bigcap\left\{\left(I_{\langle M\rangle}\right)_{v} \mid M \in t\right.$-Max $\left.(R)\right\}$.

Lemma 2.6. Let $A$ and $B$ be semiregular $w$-ideals of $R$. Then $A=B$ if and only if $A_{\langle M\rangle}=B_{\langle M\rangle}$ for each semiregular maximal $w$-ideal $M$.

Proof. Let $x \in A$ and let $I=\{r \in R \mid r x \in B\}$. It is easy to show that $I$ is a semiregular $w$-ideal of $R$ and $I \nsubseteq M$ for each semiregular maximal $w$-ideal $M$, so $I=R$. Hence we can get $x \in B$, which implies $A \subseteq B$. We can prove $B \subseteq A$ in the same way.

Theorem 2.7. Let $R$ be a ring in which each semiregular ideal is contained in at most finitely many maximal w-ideals.

(1) If for each semiregular maximal $w$-ideal $M, R_{\langle M\rangle}$ satisfies $A C C$ on semiregular $M$-reducible ideals, then $R$ satisfies $A C C$ on semiregular $w$-ideals. 
(2) If for each semiregular maximal $w$-ideal $M, R_{\langle M\rangle}$ satisfies the restricted $D C C$ on semiregular $v$-ideals, then $R$ satisfies the restricted DCC on semiregular $v$-ideals.

Proof. (1) Let $I_{1} \subseteq I_{2} \subseteq \cdots \subseteq I_{n} \subseteq \cdots$ be a chain of semiregular $w$-ideals of $R$ and let $M_{1}, M_{2}, \ldots, M_{t}$ be the semiregular maximal $w$-ideals that contain $I_{1}$. For each $M_{i},\left(I_{1}\right)_{\left\langle M_{i}\right\rangle} \subseteq\left(I_{2}\right)_{\left\langle M_{i}\right\rangle} \subseteq \cdots \subseteq\left(I_{n}\right)_{\left\langle M_{i}\right\rangle} \subseteq \cdots$ is a chain of semiregular $M_{i}$-reducible ideals of $R_{\left\langle M_{i}\right\rangle}$. Thus there is an integer $n_{i}$ such that $\left(I_{n_{i}}\right)_{\left\langle M_{i}\right\rangle}=\left(I_{k}\right)_{\left\langle M_{i}\right\rangle}$ for each $k \geq n_{i}$. Let $n=\operatorname{Max}\left\{n_{1}, n_{2}, \ldots, n_{t}\right\}$. Then for each $M_{i}$ and $k \geq n,\left(I_{n}\right)_{\left\langle M_{i}\right\rangle}=\left(I_{k}\right)_{\left\langle M_{i}\right\rangle}$. For all other semiregular maximal $w$-ideals $M$, we have $\left(I_{n}\right)_{\langle M\rangle}=R_{\langle M\rangle}=\left(I_{k}\right)_{\langle M\rangle}$, so $\left(I_{n}\right)_{\langle M\rangle}=\left(I_{k}\right)_{\langle M\rangle}$ for each semiregular maximal $w$-ideal $M$. Hence $I_{n}=I_{k}$ by Lemma 2.6.

(2) Let $I_{1} \supseteq I_{2} \supseteq \cdots \supseteq I_{n} \supseteq \cdots$ be a chain of semiregular $v$-ideals of $R$ with $\bigcap I_{n}$ semiregular and let $I=\bigcap I_{n}$. Then $I$ is contained in only finitely many semiregular maximal $w$-ideals. As above let $M_{1}, M_{2}, \ldots, M_{t}$ denote these ideals. For each $M_{i},\left(\left(I_{1}\right)_{\left\langle M_{i}\right\rangle}\right)_{v} \supseteq\left(\left(I_{2}\right)_{\left\langle M_{i}\right\rangle}\right)_{v} \supseteq \cdots \supseteq\left(\left(I_{n}\right)_{\left\langle M_{i}\right\rangle}\right)_{v} \supseteq \cdots$ is a chain of semiregular $v$-ideals of $R_{\left\langle M_{i}\right\rangle}$ with $\bigcap\left(\left(I_{n}\right)_{\left\langle M_{i}\right\rangle}\right)_{v}$ semiregular. In each ring $R_{\left\langle M_{i}\right\rangle}$ there is an integer $m_{i}$ such that $\left(\left(I_{m_{i}}\right)_{\left\langle M_{i}\right\rangle}\right)_{v}=\left(\left(I_{k}\right)_{\left\langle M_{i}\right\rangle}\right)_{v}$ for each $k \geq m_{i}$. Let $m=\operatorname{Max}\left\{m_{1}, m_{2}, \ldots, m_{t}\right\}$. Then $\left(\left(I_{m}\right)_{\left\langle M_{i}\right\rangle}\right)_{v}=\left(\left(I_{k}\right)_{\left\langle M_{i}\right\rangle}\right)_{v}$ for each $k \geq m$. For all other semiregular maximal $w$-ideals $N$ we have $\left(\left(I_{m}\right)_{\langle N\rangle}\right)_{v}=R_{\langle N\rangle}=\left(\left(I_{k}\right)_{\langle N\rangle}\right)_{v}$. Since semiregular maximal $w$-ideals are equal to semiregular maximal $t$-ideals, $I_{k}=I_{m}$ by Lemma 2.5(1).

\section{3. $Q_{0}-\mathrm{SM}$ rings}

In this section, we need the concept of finite-type $R$-modules in [7]. Now, we introduce this concept. Let $B$ and $N$ be $R$-modules and let $f \in \operatorname{Hom}_{R}(B, N)$. If for each maximal $w$-ideal $M$ of $R, f_{M}: B_{M} \rightarrow N_{M}$ is a monomorphism (resp., an epimorphism, an isomorphism), we call $f$ a $w$-monomorphism (resp., a $w$-epimorphism, a $w$-isomorphism). A module $B$ is said to be of finite type if there exist a finitely generated free module $F$ and a $w$-epimorphism $f: F \rightarrow B$ [7, Definition 1.3]. If $B$ is a GV-torsion-free module, then $B$ is of finite type if and only if there is a finitely generated submodule $A$ of $B$ such that $B_{w}=A_{w}$ [7, Proposition 1.2] and a fractional ideal $I$ is said to be a $t$-finite-type fractional ideal (resp., $v$-finite-type fractional deal) if there is a finitely generated fractional ideal $J$ contained in $I$ such that $I_{t}=J_{t}$ (resp., $I_{v}=J_{v}$ ).

The following is an SM domain analogue whose proof is similar to that of the domain case.

Lemma 3.1. The following are equivalent for $R$.

(1) $R$ satisfies $A C C$ on semiregular $w$-ideals.

(2) Each semiregular $w$-ideal of $R$ is of finite type.

(3) Each nonempty collection of semiregular $w$-ideals of $R$ has maximal elements.

(4) Each semiregular prime $w$-ideal of $R$ is of finite type.

(5) Each semiregular ideal of $R$ is of finite type. 
Let $I$ be a $w$-ideal of $R$. If $I$ is semiregular, then $I$ is said to be a semiregular $w$-ideal. In the following we define $Q_{0}$-SM rings similarly to $Q_{0}$-Mori rings.

Definition 3.2. A $Q_{0}-\mathrm{SM}$ ring is defined to be a ring which satisfies ACC on semiregular $w$-ideals and satisfies the restricted DCC on semiregular $v$-ideals.

T. Lucas has defined $Q_{0}$-Mori rings in [5]: A $Q_{0}$-Mori ring is defined to be a ring which satisfies ACC on semiregular $v$-ideals and satisfies the restricted DCC on semiregular $v$-ideals. Since $v$-ideals are $w$-ideals, a $Q_{0}$-SM ring is a $Q_{0}$-Mori ring. Each semiregular fractional ideal of a $Q_{0}$-Mori ring is a $v$-finitetype fractional ideal ([5, Theorem 2.5 and Theorem 2.7]), so is each semiregular fractional ideal of a $Q_{0}-\mathrm{SM}$ ring. Here we also have that each semiregular ideal of a $Q_{0}-\mathrm{SM}$ ring is contained in at most finitely many semiregular maximal $w$-ideals. In order to get this result, we need to define and discuss $Q_{0}-\mathrm{H}$-rings and $Q_{0}$-TV rings. A ring $R$ is said to be a $Q_{0}-H$-ring if $I$ is a semiregular ideal of $R$ and $I^{-1}=R$, then there is some $J \in \mathrm{GV}(R)$ such that $J \subseteq I$ and a ring $R$ is said to be a $Q_{0}-T V$ ring if each semiregular $t$-fractional ideal is a semiregular $v$-fractional ideal.

The following is an H-domain analogue whose proof is similar to that of the domain case.

Lemma 3.3. The following assertions are equivalent for $R$.

(1) $R$ is a $Q_{0}$-H-ring.

(2) Each semiregular maximal w-ideal of $R$ is a $v$-ideal.

(3) Let $I$ be a semiregular ideal of $R$. If $I^{-1}=R$, then $I_{w}=R$.

Since semiregular maximal $t$-ideals are maximal $w$-ideals, we have that a $Q_{0}$-TV ring is a $Q_{0}$-H-ring by Lemma 3.3 .

Lemma 3.4. Let $I$ be a semiregular $v$-ideal of $R$ and let $\left\{B_{i}\right\}$ be a nonempty collection of $v$-ideals which contain $I$. Then $\left(\sum_{i} B_{i}^{-1}\right)_{v} \subseteq I^{-1}$.

Proof. Since $I \subseteq \bigcap_{i} B_{i}=\bigcap_{i}\left(B_{i}\right)_{v}=\left(\sum_{i} B_{i}^{-1}\right)^{-1}$, we have $\left(\sum_{i} B_{i}^{-1}\right)_{v} \subseteq$ $I^{-1}$.

Lemma 3.5. Let $R$ be a $Q_{0}-T V$ ring, $I$ be a semiregular $t$-ideal of $R$, and $M$ be a maximal $w$-ideal containing $I$. If $\left\{B_{i}\right\}$ is the nonempty collection of semiregular $t$-ideals of $R$ which contain $I$ but are not contained in $M$, then $\bigcap_{i} B_{i} \nsubseteq M$.

Proof. For $B \in\left\{B_{i}\right\}$, we have $B \nsubseteq M$. Since $M$ is a maximal $v$-ideal, we have $(M+B)_{v}=R$. Hence $M^{-1} \cap B^{-1}=R$. Let $x \in M^{-1} \backslash R$, where $x \notin B^{-1}$ for every $B \in\left\{B_{i}\right\}$. Then we have $\left(\sum_{i} B_{i}^{-1}\right)_{v} \subseteq I^{-1}$ by Lemma 3.4, so there is a finitely generated semiregular ideal $I_{0}$ contained in $I$ such that $I_{0}\left(\sum_{i} B_{i}^{-1}\right)_{v} \subseteq R$. Hence $I_{0} \sum_{i} B_{i}^{-1} \subseteq R$. Therefore $\sum_{i} B_{i}^{-1}$ is a fractional ideal of $R$. Note that $\left(\sum_{i} B_{i}^{-1}\right)_{v}=\left(\sum_{i} B_{i}^{-1}\right)_{t}$ since $R$ is a $Q_{0}$-TV ring and that $x \notin\left(\sum_{i} B_{i}^{-1}\right)_{v}=\left(\sum_{i} B_{i}^{-1}\right)_{t}$. If not, there is a finitely generated submodule $F$ contained in $\sum_{i} B_{i}^{-1}$ such that $x \in F_{v}$, so there are finitely many elements 
$B_{1}, \ldots, B_{n}$ of $\left\{B_{i}\right\}$ such that $x \in\left(\sum_{j=1}^{n} B_{j}^{-1}\right)_{v}$, that is, $x \in\left(\bigcap_{j=1}^{n} B_{j}\right)^{-1}$. Since $\bigcap_{j=1}^{n} B_{j}$ is also an element of $\left\{B_{i}\right\}$, we can get a contradiction. We have $x \notin\left(\sum_{i} B_{i}^{-1}\right)_{v}=\left(\bigcap_{i} B_{i}\right)^{-1}$ and $M^{-1} \nsubseteq\left(\bigcap_{i} B_{i}\right)^{-1}$. Hence $\bigcap_{i} B_{i} \nsubseteq M$.

Lemma 3.6. Let $R$ be a $Q_{0}-T V$ ring and let $I$ be a semiregular $t$-ideal of $R$. Then $I$ is contained in at most finitely many semiregular maximal $w$-ideals.

Proof. Let $\left\{M_{i}\right\}$ denote the collection of maximal $w$-ideals containing $I, T_{i}=$ $\bigcap_{j \neq i} M_{j}$, and $T=\sum_{i} T_{i}$ for each $i$. We can get $T_{i} \nsubseteq M_{i}$ by Lemma 3.5, so $T \nsubseteq$ $M_{i}$ for each $i$. And because $I \subseteq T$, we have $T \nsubseteq M$ for each semiregular maximal $w$-ideal $M$. It follows that $T_{t}=R$, i.e., $1 \in T_{t}$. Hencce there are finitely many elements $T_{1}, \ldots, T_{n}$ of $\left\{T_{i}\right\}$ such that $1 \in\left(\sum_{i=1}^{n} T_{i}\right)_{t}=\left(\sum_{i=1}^{n} T_{i}\right)_{v}$. Let $M_{1}, \ldots, M_{n}$ be the maximal $w$-ideals corresponding to $T_{1}, \ldots, T_{n}$. Then $M_{1}, \ldots, M_{n}$ are the all maximal $w$-ideals that contain $I$. If not, there is a maximal $w$-ideal $M_{j}$ which is not equal to each $M_{i}(i=1,2, \ldots, n)$ such that $I \subseteq M_{j}$. Then $T_{i} \subseteq M_{j}, i=1, \ldots, n$. Hence $\sum_{i=1}^{n} T_{i} \subseteq M_{j}$. Therefore $1 \in\left(\sum_{i=1}^{n} T_{i}\right)_{t} \subseteq M_{j}$, which contradicts that $M_{j} \neq R$.

Lemma 3.7. Let $R$ be a $Q_{0}-S M$ ring. Then each semiregular maximal $w$-ideal of $R$ is a maximal $v$-ideal and each semiregular ideal of $R$ is contained in at most finitely many semiregular maximal $w$-ideals.

Proof. Let $I$ be a semiregular $t$-fractional ideal of $R$. Then there is a finitely generated semiregular fractional ideal $J$ contained in $I$ such that $I_{v}=J_{v}$. Thus $I=I_{t} \subseteq I_{v}=J_{v}=J_{t} \subseteq I_{t}=I$, so $I=I_{v}$ is a $v$-fractional ideal. It follows that $Q_{0}$-SM rings are $Q_{0}$-TV rings. Hence each semiregular maximal $w$-ideal of $R$ is a maximal $v$-ideal. Because the semiregular maximal $w$-ideal containing $I$ contains $I_{t}$ and $I_{t}$ is contained in at most finitely many semiregular maximal $w$-ideals by Lemma 3.6, $I$ is contained in at most finitely many semiregular maximal $w$-ideals.

Definition 3.8. For $R$ and $S, R$ is said to be a $Q_{0 S}$-Noetherian ring if $R$ satisfies ACC on semiregular $S$-reducible ideals and satisfies the restricted DCC on semiregular $v$-ideals. For a prime ideal $P$ of $R$ and $S=R \backslash P$, we say that $R$ is a $Q_{0 P^{-}}$Noetherian ring if $R$ is a $Q_{0 S^{-}}$Noetherian ring.

Next we use the $Q_{0}$-quotient $\operatorname{ring} R_{\langle S\rangle}$ with respect to $S$ to describe $Q_{0}$-SM rings.

Theorem 3.9. Let $P$ be a prime $w$-ideal of $R$. If $R$ is a $Q_{0}-S M$ ring, then $R_{\langle P\rangle}$ is a $Q_{0 P}$-Noetherian ring.

Proof. It is clear that $R_{\langle P\rangle}$ satisfies ACC on semiregular $P$-reducible ideals by Theorem 2.4.

Next we need to prove that $R_{\langle P\rangle}$ satisfies the restricted DCC on semiregular $v$-ideals.

Let $J$ be a semiregular $v$-ideal of $R_{\langle P\rangle}$ and let $A=J \cap R$. We first show that $A$ is a semiregular $v$-ideal of $R$. We can prove that $A$ is a semiregular 
ideal of $R$ in the same way as in Theorem 2.4. Because each semiregular ideal of a $Q_{0}-\mathrm{SM}$ ring is a $v$-finite-type ideal, there is a finitely generated semiregular ideal $C$ contained in $A$ such that $C_{v}=A_{v}$. We may assume $C=\left(c_{1}, c_{2}, \ldots, c_{n}\right)$. Let $u \in\left(R_{\langle P\rangle}: J\right)$. Then $u J \subseteq R_{\langle P\rangle}$. Since $C \subseteq A \subseteq J$, there is some $s \in R \backslash P$ such that $u c_{i} s \in R$ for each $i(i=1,2, \ldots, n)$. Then $u s \subseteq(R: C)=(R: C)_{v}=\left(R: C_{v}\right)=\left(R: A_{v}\right)$. Thus us $A_{v} \subseteq R$, which implies $u A_{v} \subseteq R_{\langle P\rangle}$. We have $\left(R_{\langle P\rangle}: J\right) A_{v} \subseteq R_{\langle P\rangle}$. Therefore $A_{v} \subseteq J \cap R=A$ and $A \subseteq A_{v}$ is obvious. Hence $A=A_{v}$, and so is a $v$-ideal of $R$.

To complete the proof, we need to show that $J=A_{\langle P\rangle}$. For $x \in A_{\langle P\rangle}$, we have $x s \in A \subseteq J$ for some $s \in R \backslash P$. Then $x s\left(R_{\langle P\rangle}: J\right) \subseteq R_{\langle P\rangle}$. Thus $x\left(R_{\langle P\rangle}: J\right) \subseteq R_{\langle P\rangle}$, which implies $x \in J_{v}=J$. So $A_{\langle P\rangle} \subseteq J$. The containment $J \subseteq A_{\langle P\rangle}$ is clear by Lemma 2.2(1). Thus we have $A_{\langle P\rangle}=J$.

Let $J_{1} \supseteq J_{2} \supseteq \cdots \supseteq J_{n} \supseteq \cdots$ be a descending chain of semiregular $v$ ideals of $R_{\langle P\rangle}$ with $\bigcap J_{n}$ semiregular and let $A_{i}=J_{i} \cap R$ for each $i$. By the proof above, $J_{1} \cap R \supseteq J_{2} \cap R \supseteq \cdots \supseteq J_{n} \cap R \supseteq \cdots$ is a descending chain of semiregular $v$-ideals of $R$ with $\bigcap\left(J_{n} \cap R\right)$ semiregular. Thus there is an integer $n$ such that $J_{k} \cap R=J_{n} \cap R$ for each $k \geq n$, i.e., $A_{k}=A_{n}$. We have $J_{k}=\left(A_{k}\right)_{\langle P\rangle}=\left(A_{n}\right)_{\langle P\rangle}=J_{n}$. Then we have that $\left\{J_{n}\right\}$ stabilizes. Hence $R_{\langle P\rangle}$ is a $Q_{0 P}$-Neotherian ring.

Corollary 3.10. $R$ is a $Q_{0}-S M$ ring if and only if each semiregular ideal of $R$ is contained in at most finitely many semiregular maximal $w$-ideals and $R_{\langle M\rangle}$ is a $Q_{0 M}$-Noetherian ring for each semiregular maximal $w$-ideal $M$.

Proof. The sufficiency follows from Theorem 2.7, while the necessity follows from Lemma 3.7 and Theorem 3.9.

\section{Examples}

We know that an SM domain is a Mori domain, but the converse does not hold. It is also known that a $Q_{0}-\mathrm{SM}$ ring is a $Q_{0}$-Mori ring. The first example is provided to show that the converse of this fact does not hold yet. The last two examples are provided to show the difference between $Q_{0}-\mathrm{SM}$ rings and SM rings.

Let $D$ be a domain with the quotient field $K, \mathcal{P}$ be a nonempty set of prime ideals of $D$ where $P_{\alpha}^{-1} \neq D$ for each $P_{\alpha} \in \mathcal{P}$, and $K_{\alpha}$ be the quotient field of $D / P_{\alpha}$. Then $B=\bigoplus K_{\alpha}$ is a $D$-module. Let $R=D(+) B$ be the idealization of $B$ in $R$, where for each $r, s \in D$ and $a, b \in B,(r, a)+(s, b)=(r+s, a+b)$ and $(r, a)(s, b)=(r s, a s+r b)$ (see [1, Section 25]). Let

$\mathcal{F}=\{J \mid J$ is an ideal of $D$ and for some finite subset $A$ of $J$, $A \nsubseteq P_{\alpha}$ for each $\left.P_{\alpha} \in \mathcal{P}\right\}$,

$\mathcal{F}_{0}$ be the collection of finitely generated ideals in $\mathcal{F}$, and $E=\left\{x \in K \mid x A \subseteq D\right.$ for some $\left.A \in \mathcal{F}_{0}\right\}$. 
Lemma 4.1. Let the notation be as above. Then the following hold.

(1) $(r, b) \in Z(D(+) B)$ if and only if $r \in P_{\alpha}$ for some $P_{\alpha} \in \mathcal{P}$.

(2) $I$ is a semiregular ideal of $R$ if and only if $I=I_{0}(+) B=I_{0} R$ where $I_{0} \in \mathcal{F}$.

(3) If $I=I_{0}(+) B$ is a semiregular ideal of $R$, then $\operatorname{Hom}_{R}(I, R) \cong I_{0}^{-1}(+) B$.

(4) $Q_{0}(R)=E(+) B$.

(5) $J \in G V(R)$ if and only if $J=J_{0}(+) B$ where $J_{0} \in G V(D)$.

(6) If $I$ is a semiregular ideal of $R$, then $I_{W}=\left(I_{0}\right)_{w}(+) B$ where $I_{0} \in \mathcal{F}$.

(7) If $D$ is an $\mathrm{SM}$ domain, then $R=D(+) B$ is a $Q_{0}$-SM ring.

Proof. (1) Let $r \in D$ and $P_{\alpha} \in \mathcal{P}$. If $r \notin P_{\alpha}$, then $r K_{\alpha}=K_{\alpha}$. If $r \in P_{\alpha}$, then $r c_{\alpha}=0$ for each $c_{\alpha} \in K_{\alpha}$. Hence if $r \in P_{\alpha}$, then $(r, b) \in Z(D(+) B)$ for each $b \in B$. Conversely, since $D$ is a domain, if $(r, b) \in Z(D(+) B)$, then $r$ must be an element of some $P_{\alpha} \in \mathcal{P}$.

(2), (3) See [3, Theorem 11].

(4) See [3, Theorem 11] and [4, Theorem 3].

(5) Let $J \in \mathrm{GV}(R)$. Then $J$ is a finitely generated semiregular ideal of $R$ and $J=J_{0}(+) B=J_{0} R$ where $J_{0} \in \mathcal{F}$ by $(2)$. Since $J \in \mathrm{GV}(R), \operatorname{Hom}_{R}(J, R) \cong R$. By $(3), \operatorname{Hom}_{R}(J, R) \cong J_{0}^{-1}(+) B$. So $J_{0}^{-1} \cong D$. Since $J$ is finitely generated, $J_{0}$ is a finitely generated ideal of $D$. We have $J_{0} \in \mathrm{GV}(D)$.

Conversely, if $J_{0} \in \mathrm{GV}(D)$, then $J_{0}$ is a finitely generated ideal of $D$ and $J_{0} \in$ $\mathcal{F}_{0}$. If not, $J_{0} \subseteq P_{\alpha}$ for some $P_{\alpha} \in \mathcal{P}$. Then $P_{\alpha}^{-1} \subseteq J_{0}^{-1}=D$. Hence $P_{\alpha}^{-1}=D$, which is a contradiction to the choice of $P_{\alpha}$, so we have $J=J_{0} R=J_{0}(+) B$ is a finitely generated ideal of $R$ and $J=J_{0} R=J_{0}(+) B$ is a finitely generated semiregular ideal of $R$ by (2). By (3), $\operatorname{Hom}_{R}(J, R) \cong J_{0}^{-1}(+) B=D(+) B=R$. So $J \in \mathrm{GV}(R)$.

(6) Let $I$ be a semiregular ideal of $R$. Then $I=I_{0}(+) B$ where $I_{0} \in \mathcal{F}$. Let $x \in I_{W}$. Then there is some $J \in \mathrm{GV}(R)$ such that $x J \subseteq I$ where $J=J_{0}(+) B$ and $J_{0} \in G V(D)$. Because $I_{W} \subseteq\left(Q_{0}(R)\right)_{W}=Q_{0}(R)=E(+) B \subseteq K(+) B$, $x$ can be written as $x=(e, b)$ where $e \in E, b \in B$. So $e J_{0} \subseteq I_{0}$. We have $x \in\left(I_{0}\right)_{w}(+) B$. Conversely, if $x \in\left(I_{0}\right)_{w}(+) B \subseteq D_{w}(+) B=D(+) B, x$ can be written as $x=(d, b)$ where $d \in D$ and $b \in B$, so there is some $J_{0} \in \mathrm{GV}(D)$ such that $d J_{0} \subseteq I_{0}$. By (5), we have $J=J_{0}(+) B \in \mathrm{GV}(R)$. For each $\left(a_{0}, b_{0}\right) \in J$, $(d, b)\left(a_{0}, b_{0}\right)=\left(d a_{0}, b a_{0}+d b_{0}\right) \in I_{0}(+) B$. Then $(d, b) J \subseteq I_{0}(+) B$. Hence $x \in\left(I_{0}(+) B\right)_{W}=I_{W}$. Therefore $I_{W}=\left(I_{0}\right)_{w}(+) B$.

(7) Since $D$ is an SM domain, then $D$ is a Mori domain. We have $D(+) Q_{0}(B)$ $=D(+) B=R$ is a $Q_{0}$-Mori ring by [5, Theorem 4.4], so $R$ satisfies the restricted DCC on semiregular $v$-ideals. Let $I$ be a semiregular $W$-ideal of $R$. Then $I=\left(I_{0}\right)_{w}(+) B$ by (6). $D$ is an SM domain, then $D$ satisfies ACC on $w$-ideals, so $R$ satisfies ACC on semiregular $W$-ideals. Hence we have $R$ is a $Q_{0}$-SM ring.

Example 4.2. Let $D$ be a domain, not a field and $\mathcal{P}=\{0 \mid 0$ is the zero ideal of $D\}$. Then $\mathcal{F}=\{J \mid J$ is a nonzero ideal of $D\}$. If $D$ is a Mori domain but not an SM domain, we claim that $R=D(+) B$ is a $Q_{0}$-Mori ring but not a 
$Q_{0^{-}} \mathrm{SM}$ ring. Since $D$ is not an $\mathrm{SM}$ domain, there is an infinite ascending chain $\left\{I_{0 n}\right\}$ of $w$-ideals of $D$. By Lemma 4.1(2) and (6), $\left\{I_{0 n}(+) B\right\}$ is an infinite ascending chain of semiregular $w$-ideals of $R$. Thus $R$ does not satisfy ACC on semiregular $w$-ideals. Now we give a concrete example of a $Q_{0}-\mathrm{SM}$ ring. Let $D$ denote the ring of integers $\mathbb{Z}$ and $\mathcal{P}=\operatorname{Spec}(D) \backslash\{2 D\}$. Then $R=D(+) B$ is a $Q_{0}-\mathrm{SM}$ ring.

The next example is provided to show that a $Q_{0}$-SM ring is an SM ring, but the converse does not hold.

Let $K$ be a field and $\mathcal{X}=\left\{X_{n}\right\}_{n=1}^{\infty}=\left\{X_{1}, X_{2}, \ldots, X_{n}, \ldots\right\}$, where each $X_{i}$ is an indeterminate over $K$. Let $\mathcal{F}$ denote the set of finite products of elements from $\mathcal{X}$. And for each positive integer $m$, let $\mathcal{X}^{[m]}=\left\{X_{n}^{m}\right\}_{n=1}^{\infty}$.

Example 4.3 ([5, Example 3.5]). Let $D=K\left[Y, Z, \mathcal{X}^{[2]}, \mathcal{X}^{[3]}, Y \mathcal{F}, Z \mathcal{F}\right]$ and $\mathcal{P}$ denote the set of primes of $D$ which do not contain both $Y$ and $Z$. Let $R$ be the $A+B$ ring corresponding to $D$ and $\mathcal{P}$ (see[1, Section 26]). Then $R=T(R)$, so $R$ is the only regular ideal of $R$. Hence each regular ideal of $R$ is of finite type. Then $R$ is an SM ring. Since $R$ does not satisfy the restricted DCC on semiregular $v$-ideals, we have that $R$ is not a $Q_{0}$-SM ring.

The last example is provided to show that SM rings which also satisfy the restricted DCC on semiregular $v$-ideals may not be $Q_{0}$-SM rings, i.e., ACC on regular $w$-ideals does not imply ACC on semiregular $w$-ideals.

Example 4.4 ([5, Example 3.6]). Let $\mathcal{Q}=\left\{q_{i}=\frac{p_{i}+1}{2 p_{i}} \mid p_{1}<p_{2}<\cdots\right.$, each $p_{i}$ is an odd prime $\}$ and $\mathcal{T}=\left\{t \in \mathbb{Q} \mid 0 \leq t=m+a_{1} q_{1}+\cdots+a_{n} q_{n}\right.$ with $m$ and each $a_{i}$ nonnegative integers $\}$ and $\left\{W_{i}\right\}$ be a set of indeterminates indexed over the positive integers. For each integer $k \geq 0$, let $\mathcal{W}_{k}=\left\{\prod_{i>k}^{n} W_{i}^{r_{i}} \mid 0 \leq r_{i} \in \mathbb{Z}, n>\right.$ $k\}$. We assume that $1 \in \mathcal{W}_{k}$ for each $k$. And let $\mathcal{U}=\left\{U Y^{r} Z^{s} \mid U \in \mathcal{W}_{0}, r, s \in \mathcal{T}\right.$ with $r+s \geq 1\}$ and $\mathcal{V}=\left\{V Y^{q_{i}}, V Z^{q_{i}} \mid V \in \mathcal{W}_{i}, i \geq 1\right\}$. Let $D=K[Y, Z, \mathcal{U}, \mathcal{V}]$ where $K$ is a field and $\mathcal{P}$ denote the set of primes of $D$ which do not contain both $Y$ and $Z$. Let $R$ be the $A+B$ ring corresponding to $D$ and $\mathcal{P}$. Then $R=T(R)$, so $R$ is an SM ring and $R$ satisfies the restricted DCC on semiregular $v$-ideals. Let $Q=(Y, Z, \mathcal{U}) D, Q_{1}=Q+\left(Y^{q_{1}} \mathcal{W}_{1}\right) D+\left(Z^{q_{1}} \mathcal{W}_{1}\right) D, \ldots, Q_{i}=$ $Q_{i-1}+\left(Y^{q_{i}} \mathcal{W}_{i}\right) D+\left(Z^{q_{i}} \mathcal{W}_{i}\right) D$. Then the chain $Q R \subseteq Q_{1} R \subseteq Q_{2} R \subseteq \cdots$ is a proper infinite ascending chain of semiregular $v$-ideals. Hence this chain is a proper infinite ascending chain of semiregular $w$-ideals. Therefore $R$ is not a $Q_{0}-\mathrm{SM}$ ring.

\section{References}

[1] J. Huckaba, Commutative Rings with Zero Divisors, Dekker, New York, 1988.

[2] C. J. Hwang and J. W. Lim, A note on $*_{w}$-Noetherian domains, Proc. Amer. Math. Soc. 141 (2013), no. 4, 1199-1209.

[3] T. G. Lucas, Strong Prüfer rings and the ring of finite fraction, J. Pure Appl. Algebra 84 (1993), no. 1, 59-71.

[4] $\frac{}{847-872}$. The integral closure of $R(X)$ and $R\langle X\rangle$, Comm. Algebra 25 (1997), no. 3, 
[5] - The Mori property in rings with zero divisors, Rings, modules, algebras, and abelian groups, 379-400, Lecture Notes in Pure and Appl. Math., 236, Dekker, New York, 2004.

[6] J. J. Rotman, An Introduction to Homological Algebra, Academic Press, New York, 1979.

7] F. G. Wang, Finitely presented type modules and w-coherent rings, J. Sichuan Normal Univ. 33 (2010), 1-9.

[8] F. G. Wang and R. L. McCasland, On w-modules over strong Mori domains, Comm. Algebra 25 (1997), no. 4, 1285-1306.

[9] _ On strong Mori domains, J. Pure Appl. Algebra 135 (1999), no. 2, 155-165.

[10] H. Y. Yin, F. G. Wang, X. S. Zhu, and Y. H. Chen, w-modules over commutative rings, J. Korean Math. Soc. 48 (2011), no. 1, 207-222.

Dechuan Zhou

College of Mathematics and Software Science

SiCHUAN Normal UNIVERSITY

Chengdu 610068, P. R. China

E-mail address: dechuan11119@sina.com

FANGGUI WANG

College of Mathematics and Software Science

Sichuan Normal University

Chengdu 610068, P. R. China

E-mail address: wangfg2004@163.com 